\title{
A Comparative Evaluation of Fracture Resistance of Endodontically Treated Teeth Restored By Different Post System - An Vitro Study
}

\author{
Dr Charu Dayal $^{1}$, Dr Anil Dhingra $^{2}$, Dr Neetika $^{3}$ \\ ${ }^{1}$ Department of conservative Dentistry and Endodontics, D.J. College of dental sciences \& research, India \\ ${ }^{2}$ Department of conservative Dentistry and Endodontics, D.J. College of dental sciences \& research, India \\ ${ }^{3}$ Department of conservative Dentistry and Endodontics, D.J. College of dental sciences \& research, India
}

\begin{abstract}
:
Objectives: The purpose of this in vitro study was to evaluate the fracture resistance of endodontically treated teeth restored by different post systems.

Method: Forty-Five maxillary canines with, anatomically similar root segments were taken and then decoronated at the cementoenamel-junction. After establishing the working length $1 \mathrm{~mm}$ short of the apex, the canal was prepared by crown down technique using rotary protaper followed by obturation. After 24 hours, post space preparation was done using Pesso reamer. All the specimens were then being divided into 3 groups \{Group 1 : Glass Fiber Posts, Group 2 : Carbon Fiber Posts, Group 3: Zirconia Posts\}. The posts were then cemented into the tooth using a resin sealer and acrylic resin cylinders were obtained using cylindrical molds. Specimens were subjected to increasingcompressive load $(N)$ until fracture.

Results: There was significant difference between all the three groups i.e. (Group 1: Glass fiber post, Group 2: Carbon fiber post, Group 3: Zirconia post).

Conclusion: Within the limitation of this study it was concluded that the Zirconia posts has the maximum fracture resistance followed by Glass fiber posts and Carbon fiber posts.

KeyWords: Carbon Fiber Post, Endodontically Treated Teeth , Fracture Resistance, Glass Fiber Post, Zirconia Post.
\end{abstract}

\section{Introduction}

Reconstruction of endodontically treated teeth is a great challenge in restorative dentistry since the tooth crown is usually totally or partially lost by caries, erosion, abrasion, previous restorations, trauma or endodontic access [1].If more than half of the coronal structure has been lost, a root canal post is required to provide retention for the restoration. The main objective is monoblock restoration, i.e. achievement of a single biomechanical complex by adhesion between the tooth structure and reconstruction materials (the post, luting agent and filling material) and utilisation of materials with similar mechanical properties as the remaining dentine structure [2].

Traditionally, metal posts have been used to restore endodontically treated teeth. Increased esthetic demands as well as possible problems resulting from corrosion of posts made from non-noble alloys led to the development of tooth-colored post systems [3].Heydecke et al [4] reported that the choice of an appropriate restoration for endodontically treated teeth is guided by strength and esthetics. With recent advances in ceramic technology, all ceramic crowns have become more popular. Still on this issue about esthetics, Sorenson and Mito [5] suggested that a post-core restoration supporting a translucent all-ceramic crown should not adversely affect the esthetic qualities of the definitive restoration. On this account, new tooth-colored posts can potentially improve the esthetics of the teeth restored with dowels and cores [6].

Proper restoration of endodontically treated teeth begins with a good understanding of their physical and biomechanical properties, anatomy, and a sound knowledge of the endodontic, periodontal, restorative and occlusal principles.

The purpose of this in-vitro study was to compare the fracture resistance of endodontically treated teeth restored with different post systems.

\section{Materials And Method}

Forty-Five maxillary canines freshly extracted for periodontal reasons, with straight root canals, anatomically similar root segments, and fully developed apices, were used for this study. Soft tissue and calculus were mechanically removed from these teeth and then stored in saline solution.

The teeth were then decoronated at the cementoenamel-junction using a slow-speed diamond disc.After pulp tissue was removed, the canal lengths were visually established by placing a \#10 file into each 
root canal until the tip were visible at the apical foramen. The working lengths were established $1 \mathrm{~mm}$ short of the apex. The canal was prepared by Crown Down technique using rotary Protaper (Denstply, Mallifer).

During instrumentation, canals were irrigated with 5.25\% Sodium Hypochlorite solution (Avarice Limited) and 17\% EDTA (Preverest). Before the obturation the canal was dried with paper points (Denstply, Mallifer), the obturation was performed using guttapercha (Meta Bio med) and zinc oxide eugenol sealer with lateral condensation. A radiograph of each specimen was taken to confirm satisfactory obturation of the canal Fig 1.

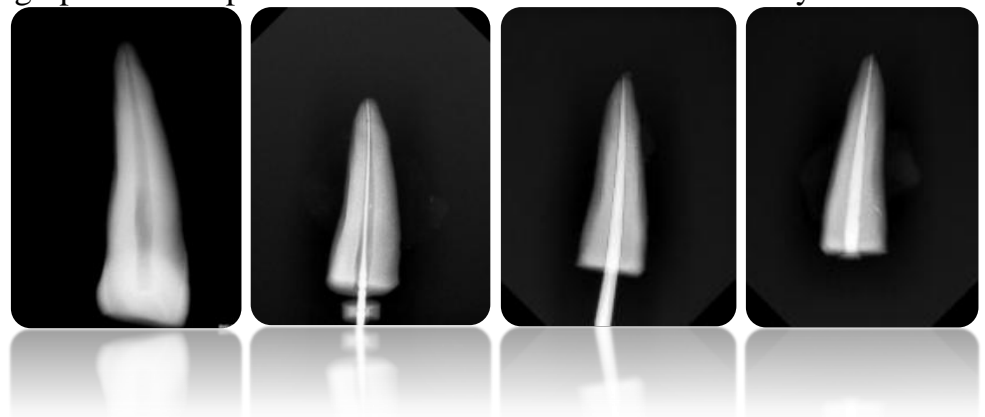

Figure 1: Radiographs of the sample (Pre-operative to Obturation)

After 24 hours, post space preparation was done using Pesso reamer, by removing guttapercha from the coronal and middle thirds of the roots leaving about $5 \mathrm{~mm}$ of intact guttapercha. Following the post space preparations the canal was irrigated with saline solution and dried with paper points (Denstply,Mallifer). Presence of any residual guttapercha in the walls of the post space was checked by radiovisiography Fig 2 .

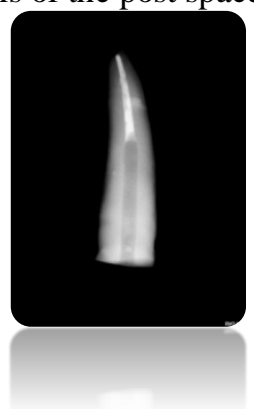

Figure 2: Radiograph after Post Space Preparation

All the specimens were then being divided into 3 groups :-
a. Group 1 : Glass Fiber Posts $(\mathrm{GF})$
b. Group 2: Carbon Fiber Posts (CF)
c. Group 3: Zirconia Posts (ZP)

The posts were then cemented into the tooth using a resin sealer (Multilink Automix-Ivoclarvivadent). The post cemented roots were then stored in saline solution at room temperature and acrylic resin cylinders were obtained using cylindrical molds.

Then the specimens were mounted on the lower plate of the Universal Testing Machine and a compressive loading was applied vertically to the coronal surfaces of the roots with a loading rate of $1 \mathrm{~mm} / \mathrm{min}$ until fracture occurred and the load at which failure has occurred was recorded and expressed in Newton.

\section{Statistical Analysis}

Descriptive statistics, including the mean, standard deviation, standard error were calculated for each of the groups tested. The results obtained were then evaluated using Bonferroni Post Hoc Test.For analyze differences within groups, the Friedman one-way ANOVA, was carried. Significance for all statistical tests was predetermined at $\mathrm{P}<.05$.

\section{Result}

The results from the experimental groups are shown in Table 1.Statistical analysis revealed that group 3 (Zirconia posts ) had more fracture resistant than other tested groups. 
TABLE 1:Mean And Standard Deviation Values

\begin{tabular}{|c|c|c|}
\hline Groups & Mean & Standard Deviation \\
\hline Glass Fibre Post & 355.87 & 29.674 \\
\hline Carbon Fiber Post & 267.60 & 13.405 \\
\hline Zirconia Post & 501.47 & 13.233 \\
\hline
\end{tabular}

TABLE 2 : Post Hoc Bonferroni Test

\begin{tabular}{|c|c|c|c|c|}
\hline Group & Comparison Group & Mean Difference & P Value & Significance \\
\hline \multirow{2}{*}{ Glass Fiber Post } & Carbon Fiber Post & 88.267 & .000 & $\mathrm{~S}^{*}$ \\
\cline { 2 - 5 } & Zirconia Post & -145.600 & .000 & $\mathrm{~S}^{*}$ \\
\cline { 2 - 5 } & Glass Fiber Post & -88.267 & .000 & $\mathrm{~S}^{*}$ \\
\cline { 2 - 5 } & Zirconia Post & -233.867 & .000 & $\mathrm{~S}^{*}$ \\
\hline Zirconia Post & Glass Fiber Post & 145.600 & .000 & $\mathrm{~S}^{*}$ \\
\cline { 2 - 5 } & Carbon Fiber Post & 233.867 & $\mathrm{~S}^{*}$ \\
\hline
\end{tabular}

$\mathrm{S}=$ Signifcant

There was significant difference between all the three groups i.e. (Group 1: Glass fiber post, Group 2: Carbon fiber post, Group 3: Zirconia post).

\section{Discussion}

Dentistry has undergone a significant evolution since its beginnings. Many technological advances have taken place since the first extracting theories. Today, the tendency is to keep any tooth, even if only a small piece remains. This is possible because of advances in endodontics, which allow the tooth to be kept once it is devitalized, and advances in restorative dentistry, with its modern restoring techniques [7].

However, endodontically treated teeth are generally weakened as a result of structure loss due to decay, previous restorative procedures and endodontic access preparation. To prevent further destruction of these teeth, a protective restoration is necessary to create retention and resistance [8]. Therefore an increased emphasis on the maintenance and preservation of natural dentition combined with an increase in the predictability and effectiveness of endodontic therapy has made their post endodontic restoration a great challenge.

Endodontic treatment removes vital contents of the canal leaving the tooth pulpless and resulting in teeth with calcified tissues that contain significantly less moisture than vital teeth. It is the manipulation of the pulp chamber that leads to the weakening of an endodontically treated tooth.

The roof of the pulp chamber has the configuration of an arch, which is extremely resistant to pressure and stress. When roof of the pulp chamber is removed for endodontic access, the inherent resistance of the endodontically treated tooth is greatly reduced. The loss of tooth structure makes retention of subsequent restorations more problematic and increases the likelihood of fracture during functional loading [9].

For years, posts have been thought to be providing reinforcement to pulpless teeth against fracture.

The primary objective of post and core procedure is replacement of the lost tooth structures in order to facilitate crown support and retention [8]. On the contrary, the tooth is weakened if dentin is sacrificed to place a large diameter dowel.

The decision regarding post placement should be based on:

$\checkmark \quad$ The amount of remaining tooth structure

$\checkmark$ Anatomic position of the tooth

$\checkmark$ Functional load on the tooth

$\checkmark$ Esthetic requirement of the tooth

In the present study Glass fiber posts, Carbon fiber posts and Zirconia posts were used.

The first fibre posts were made of carbon fibres due to their good mechanical properties. However, they were black in colour and thus lack cosmetic qualities. Although the flexural strength of fibre posts has been shown to be relatively high, large variations in the reported flexural modulus of carbon/graphite fibre posts can be found [9].

One study reported that a carbon fiber reinforced post had flexural modulus values comparable to a stainless steel post [10]. Other study [11] suggested that teeth restored with carbon fiber posts have higher fracture strengths than those with prefabricated titanium posts or cast metal post restorations.

Zirconia holds a unique place due to its superior mechanical properties and due to this it have made it a promising material for endodontic posts [9].

Hence zirconia has to be compared with a material that is both aesthetic and has good mechanical properties for use as a restorative material. One such material is Glass fibre which has been used for many years as an aesthetic post material. 
In vitro studies $[12,13]$ have shown that glass fibre posts might possess some benefits over metal posts due to their modulus of elasticity being closer to that of dentin.

In 2010 Dayalan $M$ et al [9] compared the fracture strength of the zirconia oxide posts and prefabricated glass fiber post the authors concluded that zirconium oxide posts showed higher fracture strength when compared to glass fibre posts.

The success achieved with esthetic restorative techniques has resulted in increased patient demands for these treatments, particularly for anterior teeth [14]. A non-vital anterior tooth that has lost significant tooth structure requires restoration with a crown, supported and retained by a core and possibly a post as well. Therefore in this study, maxillary canines were selected in accordance to M. Sadeghi [15] and Giovani AR et al [16].

Radio-visiography was used to determine the patency of the canal .The crowns were removed with a slow-speed diamond saw at the enamel-cement junction in accordance with Adanir N and Belli S [17].

After the post placement the teeth were loaded in an Instron Universal Testing Machine, and loading was applied to the point of fracture.

Loading to fracture represented a "worst case" scenario. Although it does not replicate what takes place in the oral environment, where teeth are subjected to forces of mastication that over a long period of time may cause fatigue resulting in tooth fracture, this method of testing has been widely used by previous researchers [18].

There are no studies \& case reports till date that compared the fracture resistance of Glass fiber post, Carbon fiber post and Zirconia post. Since these posts enable them to absorb and dissipate stresses, they were used in the present study. system used.

In the present study Zirconia posts was proven to have better fracture resistance than the other posts

Because laboratory testing cannot exactly simulate in vivo conditions, the result of any in- vitro investigation must be viewed with caution. The method evaluated in this study is technique sensitive. So, results may vary according to knowledge $\&$ experience of the operator of the technique.

\section{Conclusion}

Under the limitation of the present study, it can be concluded that, among the three posts system, used in the present study,

1. The Zirconia posts showed the maximum fracture resistance as compared to the Glass fiber posts and

Carbon fiber posts.

2. Carbon posts have the least fracture resistance.

However, long term clinical studies are required to determine the success rate of the Zirconia posts.

\section{Bibliography}

[1]. Mitsui FHO, Marchi GM, Pimenta LAF et al. In vitro study of fracture resistance of bovine roots using different intraradicular post systems. Quintessence Int2004 35: 612-616.

[2]. Duret B, Duret F, Reynaud M. Long-life physical property preservation and post endodontic rehabilitation with the Composipost. Comp Continuing Educ Dent 1996 17: 565-573.

[3]. Michalakis KX, Hirayama H, Sfolkos J, Sfolkos K. Light transmission of posts and cores used for the anterior esthetic region. Int J Periodontics Restorative Dent 2004;24:462-9.

[4]. Heydecke G, Butz F, Hussein A, StrubJR . Fracture strength after dynamic loading of endodontically treated teeth restored with different post-core systems. J Prosthet Dent 2002; 87: 438-445.

[5]. Sorensen JA, Mito WT. Rationale and clinical technique for esthetic restoration of endodontically treated teeth with the Cosmo Post and IPS Empress System. Quintessence Dent Technol 1998; 21: 81-90.

[6]. Sorensen JA, Engleman MJ. Ferrule design and fracture resistance of endodontically treated teeth. J Prosthet Dent 1990; 63: 529536.

[7]. A Barjau-Escribano, JL Sancho-Bru, L Forner-Navarro, PJ Rodríguez-Cervantes, A Pérez-González, FT Sánchez-Marín.Influence of Prefabricated Post Material on Restored Teeth: Fracture Strength and Stress Distribution.Operative Dent 2006, 31-1, 47-54

[8]. NecdetAdanira, Sema Belli.Stress Analysis of a Maxillary Central Incisor Restored with Different Posts. Eur J Dent 2007; 2:67-71

[9]. MalathiDayalan, AbhishekJairaj, K. R. Nagaraj, Ravindra C. Savadi. An Evaluation of Fracture Strength of Zirconium Oxide Posts Fabricated Using CAD-CAM Technology Compared with Prefabricated Glass Fibre Posts.J Indian ProsthodontSoc Oct-Dec 2010; 10(4):213-218

[10]. Torbjörner A, Karlsson S, Syverud M, Hensten- Pettersen A. Carbon fiber reinforced root canal posts. Mechanical and cytotoxic properties. Eur J Oral Sci 1996;104:605-11.

[11]. Isidor F, Ödman P, Brondum K. Intermittent loading of teeth restored using prefabricated carbon fiber posts. Int J Prosthodont 1996;9:131-6.

[12]. Fokkinga WA, Kreulen CM, Bronkhorst EM, Creugers NH. Up to 17-year controlled clinical study on post-and-cores and covering crowns. J Dent. 2007:35:778-786.

[13]. P. Pfeiffer, A. Schulz, I. Nergiz\& P. Schmage. Yield strength of zirconia and glass fibre-reinforced posts. J Oral Rehab 2006 33; 70-74

[14]. Oliver Pontius,\& Jeffrey W. Hutter. Survival Rate and Fracture Strength of Incisors Restored with Different Post and Core Systems and Endodontically Treated Incisors without Coronoradicular Reinforcement.JOE 2002;Vol. 28,No. 10, 710-15 
[15]. M. Sadeghi. A Comparison of the Fracture Resistance ofEndodontically Treated Teeth using Three Different Post Systems. J Dent 2006; Vol. 3, No. 2; 69-76

[16]. Alessandro RogérioGiovani, LuizPascoalVansan, ManoelDamião de Sousa Neto, and Silvana Maria Paulino. In vitro fracture resistance of glassfiber and cast metal posts with different lengths.J Prosthet Dent 2009;101:183-188

[17]. NecdetAdanira, Sema Belli.Evaluation of Different Post Lengths' Effect on Fracture Resistance of a Glass Fiber Post System. Eur J Dent 2008;2:23-28

[18]. Omar Ahmed Abo El-Ela, Osama Abdallah Atta, Omar El-Mowafy.Fracture Resistance of Anterior Teeth Restored with a Novel Non-metallic Post. JCDA 2008, Vol. 74, No. 5; 441a-441e 